\title{
Prognostic factors in stage IV gastric cancer: univariate and multivariate analyses
}

\author{
Yoshinori Yagi, Akiyoshi Seshimo, and Shingo Kameoka \\ Second Department of Surgery, Tokyo Women's Medical University, 8-1 Kawadacho, Shinjuku-ku, Tokyo 162-0054, Japan
}

\begin{abstract}
Background. The prognosis of stage IV gastric cancer is poor with the 5-year survival rate still being about $10 \%$.

Methods. We classified 130 patients with stage IV gastric cancer into four groups: peritoneal metastasis, liver metastasis, lymph node metastasis, and multiple factor groups, according to the factors that determined stage IV in each patient and compared survival in the four groups. We also performed univariate and multivariate analyses of various prognostic clinicopathological factors. The 5-year survival rate in the patients with stage IV gastric cancer was $7.4 \%$.

Results. No significant differences were observed in survival among the four groups. Univariate analysis showed significant differences in survival among the categories of lymphatic invasion $(P=0.0045)$, venous invasion $(P=0.0024)$, peritoneal metastasis $(P=0.0019)$, postoperative chemotherapy $(P=0.0385)$, curability $(P=0.0001)$, and lymph node dissection $(P=0.0001)$. In the curability $B$ group, survival was prolonged in the postoperative chemotherapy group. Multivariate analysis revealed the highest relative hazard (RH) for lymph node dissection (RH, 2.261), followed, in descending order, by curability (RH, 1.905), peritoneal metastasis (RH, 1.896), lymphatic invasion ( $R H, 1.736)$, and venous invasion (RH, 1.481).

Conclusion. As prognostic factors in stage IV gastric cancer, the tumor factors of peritoneal metastasis and vessel invasion, and the treatment factors of curability and lymph node dissection may be important, and active treatment appears to improve survival.
\end{abstract}

Key words Advanced gastric cancer - Prognostic factor · Univariate analysis $\cdot$ Multivariate analysis

\section{Introduction}

With advances in diagnostic techniques and treatment methods for gastric cancer, results of treatment for

Offprint requests to: $\mathrm{Y}$. Yagi

Received: March 2, 2000 / Accepted: June 2, 2000 gastric cancer have been improving. However, the prognosis of stage IV gastric cancer is still poor, with the 5year survival rate being about $10 \%$ [1,2]. Although all patients with stage IV gastric cancer are classified together as having advanced cancer, some of them survive for a long period. According to the first English edition of the Japanese classification of gastric carcinoma [3], the stage is determined by liver metastasis, peritoneal metastasis, lymph node metastasis, and the depth of tumor invasion. However, the prognostic factors of stage IV patients vary. To improve treatment results for stage IV gastric cancer, detailed clarification of factors associated with prognosis is important.

In recent years, there have been some studies on prognostic factors in gastric cancer by multivariate analysis. However, most of these studies evaluated gastric cancer as a whole, and none evaluated only stage IV cancer with a poor prognosis among advanced cancers.

In general, prognostic factors in gastric cancer are classified as: (1) factors in the patient (host), (2) biological factors in the tumor itself (tumor), and (3) treatment factors for the tumor (treatment) [4]. Therefore, we evaluated various factors in these three categories, by univariate and multivariate analyses, in patients with stage IV gastric cancer at our department, and analyzed the prognostic weight of the factors.

\section{Subjects and methods}

Of 1025 patients with gastric cancer encountered at our department between 1980 and 1996, 179 had stage IV cancer. Of these 179 patients, data for 130 in whom the primary lesion could be resected were analyzed in this study. The other 49 patients underwent palliative operation or only exploratory laparotomy. Stage IVa was observed in 30 patients, and stage IVb in the other 100. In the patients with stage IV gastric cancer in whom 
the primary lesion was resected, the 5-year survival rate was $7.4 \%$ (stage Iva; $12.3 \%$; stage Ivb; $6.3 \%$ ), and the $50 \%$ survival period was $205 \pm 56$ days (stage IVa, $250 \pm$ 93 days; stage IVb, $197 \pm 28$ days; mean \pm SE). No difference in survival period was observed between stages IVa and IVb $(P=0.295)$. None of the 49 patients in whom the primary lesion was not resected survived for 1 year. Compared with these patients, the patients in whom the primary lesion was resected showed a significantly longer survival $(P<0.0001)$. Of the 130 patients, 82 were men and 48 , women, and their age ranged from 25 to 87 years (mean \pm SE, $61.1 \pm 1.1$ years).

\section{Methods}

The patients were classified into four groups according to the factors that determined stage IV in each patient, using the factors for stage grouping in the first English edition of the Japanese Classification of gastric carcinoma (peritoneal metastasis, liver metastasis, lymph node metastasis - depth of invasion, and multiple factor groups) [3], and survival in the four groups was compared.

As prognostic factors, we evaluated (1) host factors; (2) factors in the primary lesion and intraperitoneal metastatic lesions, as tumor factors; and (3) treatment factors.

As host factors, age and sex were selected. As factors in the primary lesion, the following clinicopathological factors in gastric cancer, provided in the first English edition of the Japanese Classification of gastric carcinoma [3], were selected: depth of invasion, histological type, macroscopic type, location, lymphatic invasion, and venous invasion.

As factors in intraperitoneal metastases, liver metastasis, peritoneal metastasis, and lymph node metastasis were selected.

As treatment factors, curability, lymph node dissection, gastrectomy method, and postoperative chemotherapy were selected.

\section{Statistical analysis}

Statistical analysis was performed using a Power Macintosh G3 and StatView 5.0 (SAS Institute, Berkeley, CA, USA) software.

Cumulative survival curves according to the categories of each prognostic factor were obtained by the Kaplan-Meier method, and the log-rank test was used for the analysis of differences.

Prior to multivariate analysis, screening of each factor was performed by the stepwise method, and factors inappropriate for multivariate analysis were excluded. For multivariate analysis, Cox's proportional hazard model was used. The prognostic weight on multivariate analysis was ranked in terms of the relative hazard $(\mathrm{RH})$, and differences were analyzed by parameter estimate $(\beta)$, SE, $\chi 2, P$ value $(P)$, and $95 \%$ confidence interval $(95 \% \mathrm{CI})$. Analysis was discontinued at the time of death, final confirmation of survival, or on June 7, 1997. The maximum follow-up period was 5 years after operation.

Staging of gastric cancer and clinicopathological factors used in this study were based on the first English edition of the Japanese Classification of gastric carcinoma (Japanese Research Society for Gastric Cancer) [3].

\section{Results}

\section{Univariate analysis}

Evaluation according to factors that determined stage IV in each patient

The patients with stage IV gastric cancer in whom the primary lesion was resected were classified, according to the factors that determined stage IV, into the following four groups: peritoneal metastasis group (P group), liver metastasis group (L group), lymph node metastasis and depth of invasion (sewsa-infiltrating [si]) group (LN/tumor invasive depth [T] group), and multiple factor group (P and L or P and LN/T group). In this study, there was no patient in whom stage IV was determined based on $\mathrm{L}$ and $\mathrm{LN} / \mathrm{T}$ (Table 1). The 5-year survival rate was $16.2 \%$ in the $\mathrm{P}$ group $(n=46), 0 \%$ in the $\mathrm{L}$ group $(n=33), 3.6 \%$ in the LN/T group $(n=344)$, and $8.1 \%$ in the multiple factor group $(n=17)$. The $50 \%$ survival period was $193 \pm 74$ days in the $\mathrm{P}$ group, $161 \pm 26$ days in the L group, $258 \pm 23$ days in the LN/T group, and $295 \pm 143$ in the multiple factor group. No significant differences were observed in survival among the four groups $(P=0.1063)$, but survival was slightly prolonged in the $\mathrm{P}$ group (Fig. 1).

Host, tumor, and treatment factors

Table 2 shows the number of patients, the 5-year survival rates, and the $P$ value in the logrank test according to the categories of each prognostic factor.

Host factors. According to age at the time of operation, the 5-year survival rate was $4.9 \%$ for those aged less than 50 years, $12.4 \%$ for those aged $50-59$ years, $6.0 \%$ for those aged $60-69$ years, and $9.1 \%$ for those aged 70 years or more. No significant differences were observed in survival among these categories $(P=$ 0.721 ). According to sex, the 5-year survival rate was $7.4 \%$ in the men and $8.0 \%$ in the women, the difference being non-significant $(P=0.979)$. 
Table 1. Stage IV factors

\begin{tabular}{llcc}
\hline & \multicolumn{1}{c}{ Factors } & No. of patients & 5-Year survival rate \\
\hline Single factor & Peritoneal metastases & 46 & $16.2 \%$ \\
& Liver metastases & 33 & $0.0 \%$ \\
Multiple factors & Lymph node metastases/T & 34 & $3.6 \%$ \\
Total & P and L; P and LN/T & 17 & $8.1 \%$ \\
\hline
\end{tabular}

P, Perctoneal metastasis; L, liver metastasis; $\mathrm{T}$, tumor invasive depth

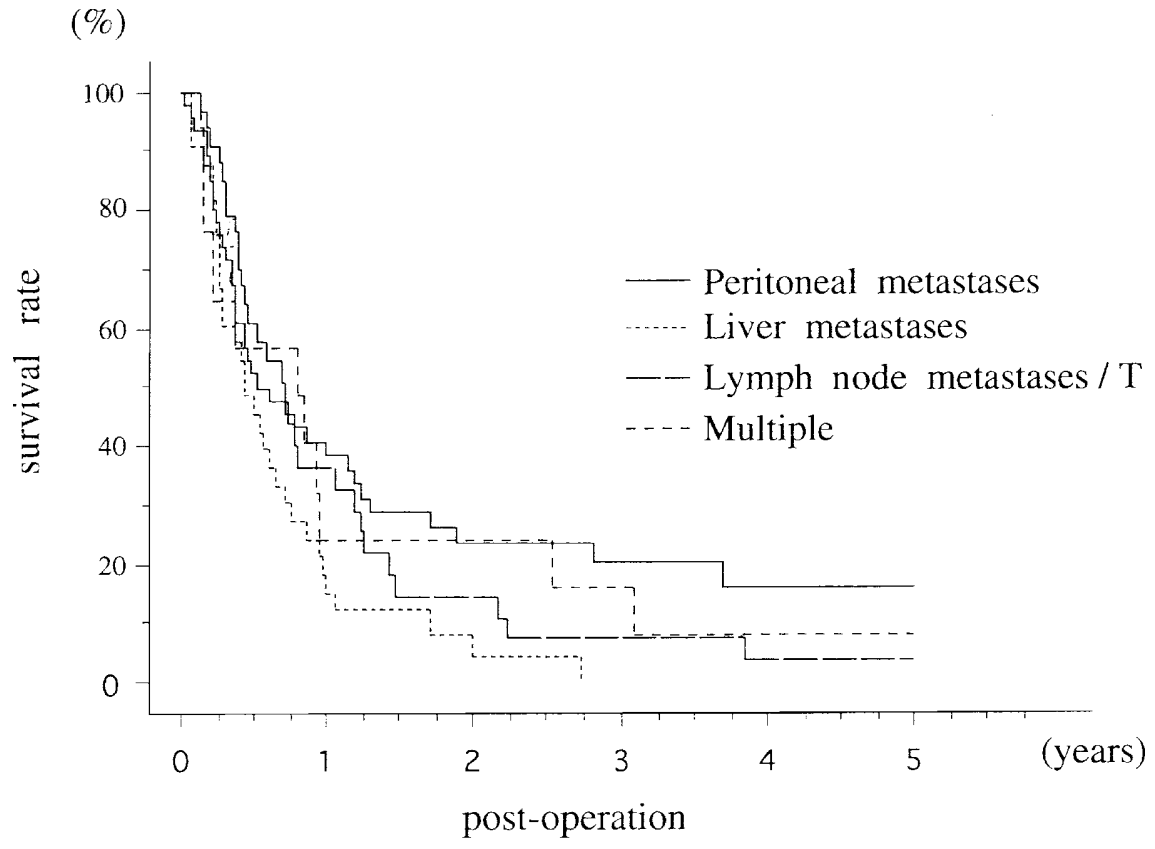

Fig. 1. Survival curves by stage IV factors. T, Tumor invasive depth

\section{Tumor factors}

Clinicopathological factors in the primary lesion. Depth of invasion. Depth of invasion was classified as muscularis propria subserosa (mp/ss), serosa exposed (se), or sewsa-infiltrazing (si). The 5-year survival rate was $12.8 \%$ for $\mathrm{mp} / \mathrm{ss}, 0.0 \%$ for $\mathrm{se}$, and $10.5 \%$ for si. No significant difference was observed in survival among the three categories $(P=0.260)$.

Histological classification. The histological type was classified as differentiated or undifferentiated. The differentiated type included papillary adenocarcinoma (pap) and well differentiated and moderately differentiated tubular adenocarcinoma (tub1 and tub2, respectively). The undifferentiated type included solid and non-solid poorly differentiated adenocarcinoma (por1 and por2, respectively), signet ring cell carcinoma (sig), and mucinous carcinoma (muc). The 5-year survival rate was $8.3 \%$ for the differentiated type and $7.1 \%$ for the undifferentiated type, the difference being nonsignificant $(P=0.081)$.

Macroscopic type. The macroscopic type was classified as localized (types 1 and 2) or diffuse (types 3, 4, and 5).
The 5-year survival rate was $21.6 \%$ for the localized type and $4.8 \%$ for the diffuse type, showing no significant difference $(P=0.141)$.

Location. The primary area where the tumor was located was classified into three areas (lower-third, middle-third, upper-third; L, M, and $\mathrm{U}$, respectively). The 5-year survival rate was $11.9 \%$ for the $\mathrm{L}$ area, $3.0 \%$ for the $\mathrm{M}$ area, and $9.0 \%$ for the $\mathrm{U}$ area, showing no differences $(P=0.2456)$.

Lymphatic invasion. Lymphatic invasion (ly) was classified as slight (ly0, ly1) or marked (ly2, ly3). The 5 -year survival rate was $25.0 \%$ for slight lymphatic invasion, but $1.4 \%$ for marked lymphatic invasion, the difference being significant $(P=0.0045)$.

Venous invasion. Venous invasion ( $\mathrm{v}$ ) was classified as slight (v0, v1) or marked (v2, v3). The 5-year survival rate was $11.6 \%$ for slight venous invasion but $0.0 \%$ for marked venous invasion, showing a significant difference $(P=0.0024)$. Evaluation was also performed using the following four combinations of the lymphatic invasion categories and the venous invasion categories: slight lymphatic invasion and slight venous invasion 
Table 2. Univariate analysis of prognostic factors

\begin{tabular}{|c|c|c|c|c|}
\hline Prognostic factor & Category & No. of patients & 5-Year survival rate $(\%)$ & $\mathrm{P}$ value $^{\mathrm{a}}$ \\
\hline \multirow[t]{2}{*}{ Sex } & Male & 82 & 7.4 & 0.9787 \\
\hline & Female & 48 & 8.0 & \\
\hline \multirow[t]{4}{*}{ Age (years) } & $\leqslant 49$ & 26 & 4.9 & 0.7214 \\
\hline & $50-59$ & 23 & 12.4 & \\
\hline & $60-69$ & 44 & 6.0 & \\
\hline & $\geqslant 70$ & 37 & 9.1 & \\
\hline \multirow[t]{3}{*}{ Depth of invasion ${ }^{\mathrm{b}}$} & $\mathrm{mp}, \mathrm{ss}$ & 48 & 12.8 & 0.2599 \\
\hline & se & 58 & 0.0 & \\
\hline & si & 24 & 10.5 & \\
\hline \multirow[t]{2}{*}{ Histological type ${ }^{b}$} & pap, tub1,2 & 42 & 8.3 & 0.0814 \\
\hline & por1,2, sig, muc & 88 & 7.1 & \\
\hline \multirow[t]{2}{*}{ Macroscopic type } & Localized & 26 & 21.6 & 0.1410 \\
\hline & Diffuse & 104 & 4.8 & \\
\hline \multirow[t]{3}{*}{ Location } & Lower-third & 43 & 11.9 & 0.2456 \\
\hline & Middle-third & 58 & 3.0 & \\
\hline & Upper-third & 29 & 9.0 & \\
\hline \multirow[t]{2}{*}{ Lymphatic invasion $^{\mathrm{b}}$} & ly0 0,1 & 30 & 25.0 & 0.0045 \\
\hline & ly 2,3 & 100 & 1.4 & \\
\hline \multirow[t]{2}{*}{ Venous invasion ${ }^{\mathrm{b}}$} & v0,1 & 84 & 11.6 & 0.0024 \\
\hline & $\mathrm{v} 2,3$ & 46 & 0.0 & \\
\hline \multirow[t]{4}{*}{ Peritoneal metastases } & P0 & 67 & 1.9 & 0.0019 \\
\hline & $\mathrm{P} 1$ & 33 & 20.4 & \\
\hline & $\mathrm{P} 2$ & 18 & 13.0 & \\
\hline & $\mathrm{P} 3$ & 12 & 0.0 & \\
\hline \multirow[t]{4}{*}{ Liver metastases } & $\mathrm{H} 0$ & 88 & 10.0 & 0.1904 \\
\hline & $\mathrm{H} 1$ & 15 & 0.0 & \\
\hline & $\mathrm{H} 2$ & 14 & 7.0 & \\
\hline & $\mathrm{H} 3$ & 13 & 0.0 & \\
\hline \multirow[t]{3}{*}{ Lymph node metastases } & $\mathrm{n} 0,1$ & 41 & 12.8 & 0.2256 \\
\hline & $\mathrm{n} 2$ & 49 & 6.4 & \\
\hline & $\mathrm{n} 3,4$ & 40 & 3.4 & \\
\hline \multirow[t]{2}{*}{ Curability } & B & 27 & 17.5 & 0.0001 \\
\hline & $\mathrm{C}$ & 103 & 5.0 & \\
\hline \multirow[t]{2}{*}{ Lymph node dissection ${ }^{\mathrm{b}}$} & D0,1 & 49 & 2.3 & $<0.0001$ \\
\hline & $\mathrm{D} 2,3,4$ & 81 & 11.3 & \\
\hline \multirow[t]{2}{*}{ Gastrectomy method } & TGR & 74 & 6.7 & 0.4647 \\
\hline & STGR & 54 & 8.6 & \\
\hline \multirow[t]{2}{*}{ Postoperative chemotherapy } & Presence & 44 & 14.7 & 0.0385 \\
\hline & Absence & 86 & 2.85 & \\
\hline
\end{tabular}

TGR, Total gastrectomy; STGR, subtotal gastrectomy

${ }^{\text {a }} \mathrm{P}$ value evaluated by Log-rank test

${ }^{\mathrm{b}}$ See text for definitions

(ly0, ly1 $\cdot v 0, v 1)$, slight lymphatic invasion and marked venous invasion (ly0, ly1 · v2, v3), marked lymphatic invasion and slight venous invasion (ly2, ly3 - v0, v1), and marked lymphatic invasion and marked venous invasion (ly2, ly3 - v2, v3). As shown in Table 3, the 1year, 3-year, and 5-year survival rates were significantly higher for slight lymphatic invasion and slight venous invasion $(\mathrm{ly} 0, \mathrm{ly} 1 \cdot \mathrm{v} 0, \mathrm{v} 1)$ than in the other three vessel invasion categories $(P=0.033, P=0.001$, and $P=$ $0.0004)$. In addition, the association with clinicopathological factors was compared between the group with slight lymphatic invasion and slight venous invasion and the other three groups. As shown in Table 4, the L area for location, the localized macroscopic type, and slight
Table 3. Survival rates in vessel invasion groups

\begin{tabular}{lcccc}
\hline & $\begin{array}{c}\text { Number of } \\
\text { patients }\end{array}$ & 1 Year & 3 Year & 5 Year \\
\hline ly0,1, v0,1 & 23 & $52.0 \%$ & $32.6 \%$ & $32.6 \%$ \\
ly2,3, v0,1 & 61 & $26.0 \%$ & $7.3 \%$ & $2.4 \%$ \\
ly0,1, v2,3 & 7 & $14.3 \%$ & $0.0 \%$ & $0.0 \%$ \\
ly2,3, v2,3 & 39 & $18.1 \%$ & $0.0 \%$ & $0.0 \%$ \\
$P$ value $^{\text {a }}$ & & 0.0332 & 0.0012 & 0.0004
\end{tabular}

a $P$ value evaluated by log-rank test 
Table 4. Correlation between vessel invasion and clinicopathological factors

\begin{tabular}{|c|c|c|c|c|}
\hline Prognostic factor & Category & ly0,1 and v0,1 & ly 2,3 or $v 2,3$ & $P$ value $^{\text {a }}$ \\
\hline \multirow[t]{2}{*}{ Sex } & Male & 13 & 69 & NS \\
\hline & Female & 10 & 38 & \\
\hline Age (years) & & $61.00 \pm 2.55$ & $61.16 \pm 1.25$ & NS \\
\hline \multirow[t]{3}{*}{ Depth of Invasion } & $\mathrm{mp}, \mathrm{ss}$ & 12 & 36 & NS \\
\hline & se & 5 & 53 & \\
\hline & si & 6 & 18 & \\
\hline \multirow[t]{2}{*}{ Histological type } & pap, tub1,2 & 9 & 33 & NS \\
\hline & por1,2, sig, muc & 14 & 74 & \\
\hline \multirow[t]{2}{*}{ Macroscopic type } & Localized & 9 & 17 & 0.0195 \\
\hline & Diffuse & 14 & 90 & \\
\hline \multirow[t]{3}{*}{ Location } & Lower-third & 15 & 28 & 0.0011 \\
\hline & Middle-third & 4 & 54 & \\
\hline & Upper-third & 4 & 25 & \\
\hline \multirow[t]{4}{*}{ Peritoneal metastases } & $\mathrm{PO}$ & 11 & 56 & NS \\
\hline & $\mathrm{P} 1$ & 10 & 23 & \\
\hline & $\mathrm{P} 2$ & 2 & 16 & \\
\hline & P3 & 0 & 12 & \\
\hline \multirow[t]{4}{*}{ Liver metastases } & $\mathrm{H} 0$ & 16 & 72 & NS \\
\hline & H1 & 2 & 13 & \\
\hline & $\mathrm{H} 2$ & 1 & 13 & \\
\hline & H3 & 4 & 9 & \\
\hline \multirow[t]{3}{*}{ Lymph node metastases } & n0,1 & 14 & 27 & 0.0038 \\
\hline & $\mathrm{n} 2$ & 5 & 44 & \\
\hline & $\mathrm{n} 3,4$ & 4 & 36 & \\
\hline
\end{tabular}

NS, Not significant

${ }^{a} P$ value evaluated by $\chi^{2}$ test, Fisher's test, Mann-Whitney $U$-test, or unpaired $t$-test

Table 5. Survival rates in peritoneal metastasis-positive groups

\begin{tabular}{lcrrr}
\hline & & \multicolumn{3}{c}{ Post-operation } \\
\cline { 3 - 5 } Peritoneal metastases & Number of patients & 1 Year & 3 Year & 5 Year \\
\hline P1 & 33 & $51.5 \%$ & $29.8 \%$ & $20.4 \%$ \\
P2 & 18 & $13.0 \%$ & $13.0 \%$ & $13.0 \%$ \\
P3 & 12 & $20.0 \%$ & $0.0 \%$ & $0.0 \%$ \\
P value & & 0.0100 & 0.0042 & 0.0057 \\
\hline
\end{tabular}

${ }^{\text {a }} \mathrm{P}$ value evaluated by log-rank test

lymph node metastasis (n0, n1) were less frequently observed in the slight lymphatic invasion and slight venous invasion group $(P=0.0011, P=0.0195$, and $P=$ 0.0038 , respectively).

Metastasis factors. Liver metastasis $(H)$. Liver metastasis was classified as $\mathrm{H} 0, \mathrm{H} 1, \mathrm{H} 2$, and $\mathrm{H} 3$. The 5-year survival rate was $10.0 \%$ for $\mathrm{H} 0,0.0 \%$ for $\mathrm{H} 1,7.0 \%$ for $\mathrm{H} 2$, and $0.0 \%$ for $\mathrm{H} 3$, showing no significant differences among the four categories $(P=0.190)$.

Peritoneal metastasis $(P)$. Peritoneal metastasis was classified as $\mathrm{P} 0, \mathrm{P} 1, \mathrm{P} 2$, and $\mathrm{P} 3$. The 5-year survival rate was $1.9 \%$ for $\mathrm{P} 0,20.4 \%$ for $\mathrm{P} 1,13.0 \%$ for $\mathrm{P} 2$, and $0.0 \%$ for P3, being significantly higher for P1 than for the other three categories $(P=0.002)$. Among the $\mathrm{P}$ positive categories, survival was significantly longer for P1 than for $\mathrm{P} 2$ or $\mathrm{P} 3(P=0.0057)$ (Table 5).
Lymph node metastasis (n). Lymph node metastasis was classified as $\mathrm{n} 0 / \mathrm{n} 1, \mathrm{n} 2$, and $\mathrm{n} 3 / \mathrm{n} 4$. The 5 -year survival rate was $12.8 \%$ for $\mathrm{n} 0 / \mathrm{n} 1,6.4 \%$ for $\mathrm{n} 2$, and $3.4 \%$ for $\mathrm{n} 3 /$ n4. Survival did not differ among the three categories $(P=0.2256)$.

\section{Treatment factors}

Curability. Curability was classified as B or C. The 5year survival rate was $17.5 \%$ for curability B and $5.0 \%$ for curability C. Survival was significantly longer for curability B $(P=0.0001)$.

Lymph node dissection. The degree of lymph node dissection was classified as slight (D0, D1) and marked (D2, D3, D4). The 5-year survival rate was $2.3 \%$ for slight lymph node dissection, but $11.3 \%$ for marked lymph node dissection. Survival was significantly longer 


\section{Curability B}

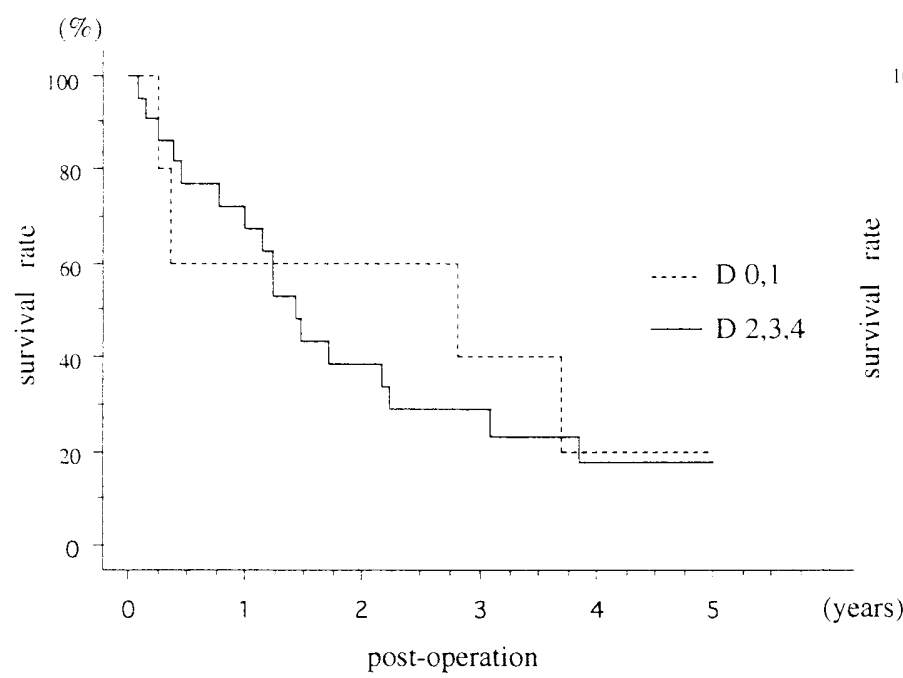

Curability $\mathrm{C}$

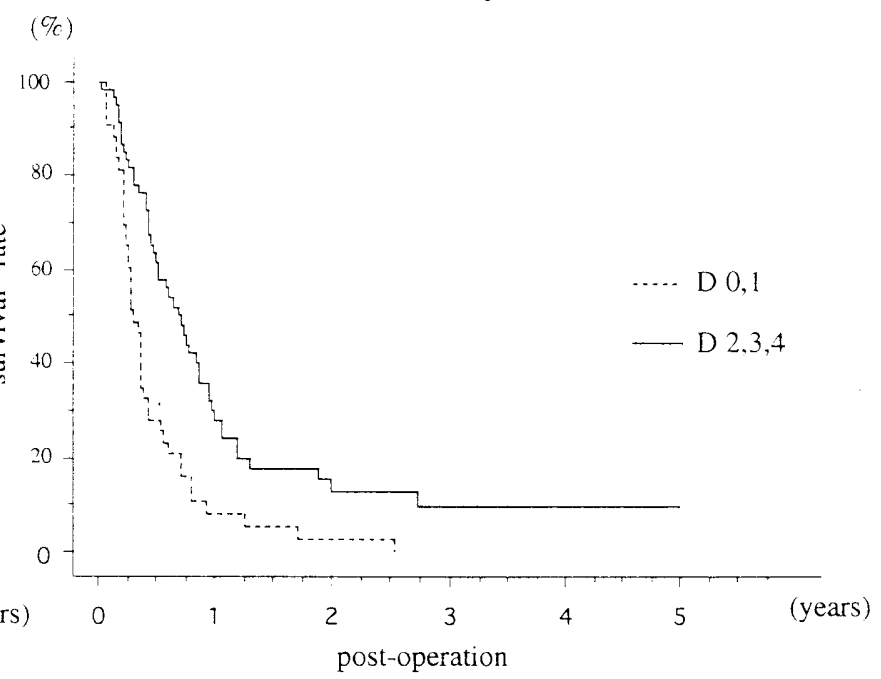

Fig. 2. Survival curves by lymph node dissection in curability B group and curability C group. D0,1, Slight lymph node dissection; D2,3,4, marked lymph node dissection

for marked lymph node dissection $(P<0.0001)$. In each curability category, survival was compared between slight and marked lymph node dissection. In curability $B$, survival did not significantly differ between slight lymph node dissection $(n=5)$ and marked lymph node dissection $(n=22)(P=0.7330)$. In curability $\mathrm{C}$, the 5year survival rate was $2.7 \%$ for slight lymph node dissection $(n=43)$ and $7.4 \%$ for marked lymph node dissection $(n=60)$, showing significantly longer survival for the latter $(P=0.0007)$ (Fig. 2$)$.

Gastrectomy method. The resection methods used for the primary lesion were classified as total gastrectomy and subtotal gastrectomy. The 5-year survival rate was $6.7 \%$ for total gastrectomy and $8.6 \%$ for subtotal gastrectomy, showing no significant difference $(P=$ 0.4647).

Postoperative chemotherapy. Postoperative chemotherapy was classified as the presence or absence of postoperative intravenous administration of anticancer drugs. The 5-year survival rate was $14.7 \%$ for the presence of postoperative chemotherapy but $2.8 \%$ for the absence of postoperative chemotherapy, showing significantly longer survival for the former group $(P=0.0385)$. In our department, 5-fluorouracil (FU) . mitomycin C (MMC) therapy, FAM therapy (5-FU, Adriamycin, MMC), or 5-FU · methotvexate (MTX) therapy has been employed as postoperative chemotherapy. No differences were observed in survival among these therapies $(P=0.580)$. In each curability category, survival was compared between the presence and absence of postoperative chemotherapy. In curabil- ity $\mathrm{B}$, the 5 -year survival rate was $31.2 \%$ for the presence of postoperative chemotherapy $(n=14)$ and $0.0 \%$ for the absence of postoperative chemotherapy, showing significantly longer survival for the former $(P=$ $0.045)$. In curability $C$, survival did not differ between the presence $(n=30)$ and absence $(n=73)$ of postoperative chemotherapy $(P=0.6898)$ (Fig. 3).

\section{Multivariate analysis}

Prior to the performance of multivariate analysis, the validity of multivariate analysis of the 15 prognostic factors was evaluated by the stepwise method. As a result, 9 factors (age, sex, depth of invasion, macroscopic type, location, liver metastasis, lymph node metastasis, postoperative chemotherapy, and gastrectomy method) were excluded. Therefore, multivariate analysis of the other 6 factors (histological type, lymphatic invasion, venous invasion, peritoneal metastasis, curability, lymph node dissection) was performed (Table 6). Of the 6 factors, lymph node dissection showed the highest relative hazard (RH) value (2.261), followed in descending order by curability (1.905), peritoneal metastasis (1.896), lymphatic invasion (1.736), venous invasion (1.481), and histological type (1.406).

\section{Discussion}

Treatment results for gastric cancer have improved markedly, but mainly in curability A patients (those with early gastric cancer). In patients with stage IV 


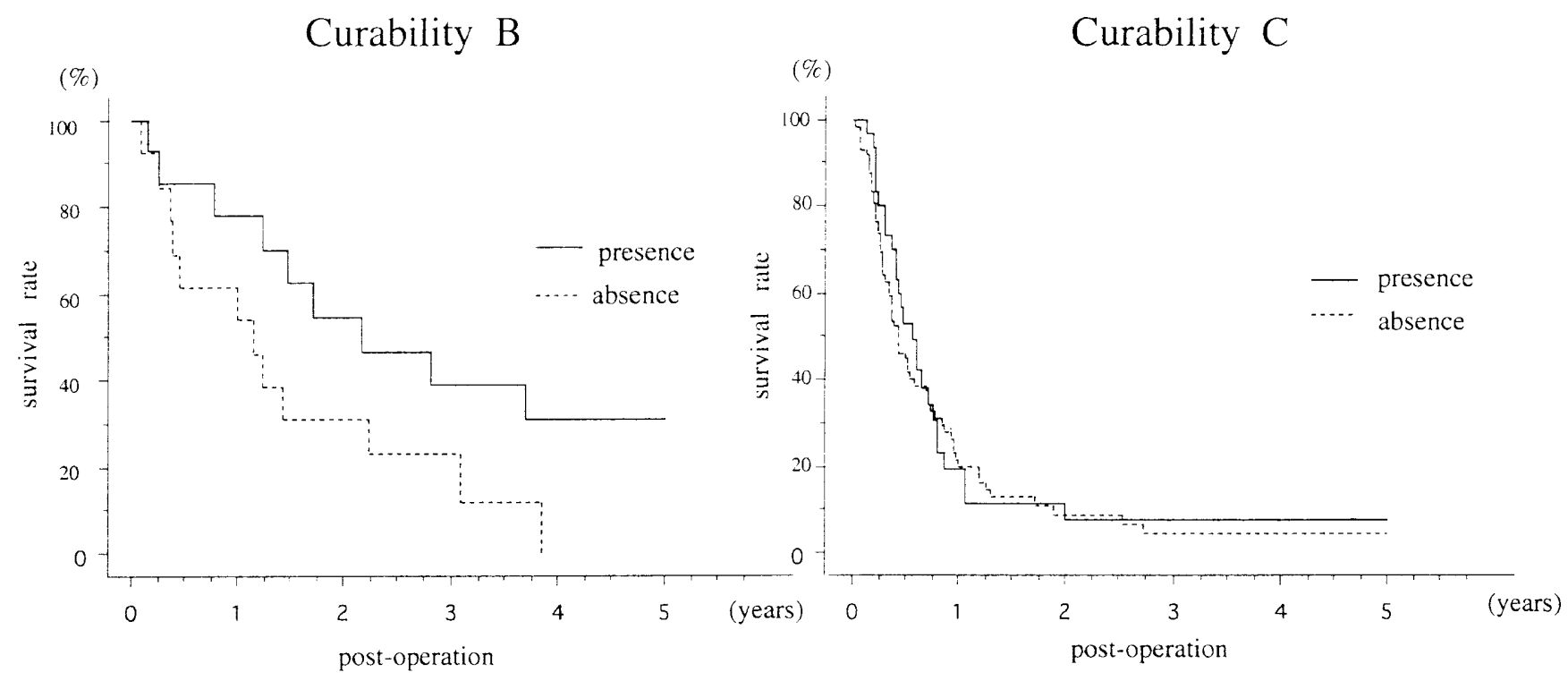

Fig. 3. Survival curves by chemotherapy in curability B group and curability C group. Presence, Presence of postoperative intravenous administration of anticancer drugs; absence, no postoperative anticancer drugs

Table 6. Multivariate analysis of prognostic factors by stepwise method (Cox's proportional hazard model)

\begin{tabular}{|c|c|c|c|c|c|c|c|}
\hline Prognostic factor & Category & RH & $95 \% \mathrm{CI}$ & $P$ value & $\beta$ & SE & $\chi^{2}$ \\
\hline \multirow{4}{*}{$\begin{array}{l}\text { Lymph node } \\
\text { dissection } \\
\text { Curability }\end{array}$} & D $2,3,4$ & 1 & & & & & \\
\hline & D 0,1 & 2.261 & $1.504-3.398$ & $<0.0001$ & 0.816 & 0.208 & 15.407 \\
\hline & B & 1 & & & & & \\
\hline & $\mathrm{C}$ & 1.905 & $1.073-3.378$ & 0.0277 & 0.644 & 0.292 & 4.849 \\
\hline \multirow{4}{*}{$\begin{array}{l}\text { Peritoneal } \\
\text { metastasis }\end{array}$} & $\mathrm{P} 1$ & 1 & & & & & \\
\hline & $\mathrm{P} 0$ & 1.896 & $1.116-3.222$ & 0.0181 & 0.640 & 0.271 & 5.590 \\
\hline & $\mathrm{P} 2$ & 1.770 & $0.860-3.636$ & 0.1214 & 0.570 & 0.368 & 2.399 \\
\hline & P3 & 2.146 & $0.967-4.762$ & 0.0604 & 0.765 & 0.407 & 3.527 \\
\hline \multirow{4}{*}{$\begin{array}{l}\text { Lymphatic } \\
\text { invasion } \\
\text { Venous invasion }\end{array}$} & $\operatorname{ly} 0,1$ & 1 & & & & & \\
\hline & ly 2,3 & 1.736 & $1.055-2.857$ & 0.0299 & 0.552 & 0.254 & 4.715 \\
\hline & v0,1 & 1 & & & & & \\
\hline & $\mathrm{v} 2,3$ & 1.481 & $0.977-2.252$ & 0.0644 & 0.394 & 0.213 & 3.420 \\
\hline \multirow[t]{2}{*}{ Histological type } & Diff. & 1 & & & & & \\
\hline & Undiff. & 1.406 & $0.923-2.141$ & 0.1121 & 0.341 & 0.214 & 2.525 \\
\hline
\end{tabular}

RH, Relative hazard; undiff., undifferentiated type; diff., differentiated type; 95\% CI, 95\% confidence interval

gastric cancer, the 5-year survival rate is still about $10 \%$ $[1,2]$. However, some patients with stage IV cancer show long survival. Therefore, we evaluated prognostic factors of gastric cancer to identify those determining the prognosis of stage IV cancer, and we also analyzed their clinical validity.

First, we evaluated possible differences in survival rates according to factors that determine stage IV in each patient. Because no differences in survival were observed among the factors determining stage IV, other clinicopathological factors were evaluated to identify those associated with the prognosis of stage IV gastric cancer.
No differences were observed in survival according to sex, as reported by previous studies, or according to age. Other studies have shown poorer prognosis with age. This is considered to be due to deaths from other diseases [5]. However, because most patients with advanced stage IV gastric cancer in all age groups die of recurrence, the percentage of those who die of other diseases may be low.

Concerning depth of invasion, the prognosis is generally poorer with deeper invasion. In particular, serous invasion-positive patients have a poorer prognosis $[1,6]$. The effects of depth of invasion on the prognosis of gastric cancer may be associated with a higher 
probability of peritoneal metastasis when serous invasion is positive. However, in stage IV gastric cancer, there are various prognostic factors other than peritoneal metastasis, such as lymph node metastasis and liver metastasis. This may be the reason for the only slightly lower survival rate in the serous invasionpositive groups in our study.

In general, the prognosis is considered to be better for well differentiated carcinoma than for undifferentiated carcinoma, but some studies have suggested no difference in prognosis between the two types $[1,6,7]$. In this study, no difference in survival was observed between the differentiated and undifferentiated types.

Concerning macroscopic type, in general, the prognosis is poorer for the diffuse type than for the localized type $[1,2,4,6]$. In this study, survival was slightly longer, but not significantly, for the localized type.

Many studies have shown the best prognosis for tumors located in the $\mathrm{M}$ area, followed in descending order by those located in the $\mathrm{L}$ area and those in the $\mathrm{U}$ area [8]. This has been suggested to be not only due to the anatomical position of the tumor but also due to differences in the pattern of progression among the areas [8]. In this study, no differences were observed in survival among the areas. Because stage IV gastric cancer is considerably advanced, differences in the pattern of progression among the areas may have been reduced.

Vessel invasion (venous invasion, lymphatic invasion) increases lymphogenous and hematogenous metastasis. Therefore, the survival rate is considered to be lower for $l \mathrm{y}(+)$ or $\mathrm{v}(+)$ than for $\operatorname{ly}(-)$ or $\mathrm{v}(-)$ [8]. Studies at other institutions have shown poorer prognosis with increasing degree of vessel invasion [1,2,9-12]. Another study reported differences in prognosis only according to lymphatic invasion [7]. In the present study, the prognosis was significantly poorer in the marked venous invasion group (v2,3) and in the marked lymphatic invasion group $(1 y 2,3)$ than in the slight invasion group. In general, the degree of vessel invasion increases with the depth of invasion, and, therefore, the association of vessel invasion with prognosis should be carefully evaluated [8]. When the two categories (slight, marked) of venous invasion were combined with those of lymphatic invasion to obtain four vessel invasion groups, survival was significantly prolonged in the slight vessel invasion group (ly0/1, v0/ 1) compared with the other groups. The association between this group and other clinicopathological factors was evaluated. In this slight vessel invasion group, the localized type and slight lymph node metastasis were less frequently observed. These findings were rational because of the mild infiltrative growth pattern. To determine whether the infiltrative growth pattern dif- fers among the areas, further studies are necessary. However, no significant difference was observed in depth of invasion between the slight vessel invasion group and the other groups. In stage IV gastric cancer, the effects of degree of vessel invasion on prognosis are worth evaluation.

Concerning intraperitoneal metastatic lesions, the survival rate differed significantly according to degree of peritoneal metastasis. Among the above factors determining stage IV, survival was slightly longer in the $P$ group. Multivariate analysis revealed the third highest prognostic weight of the $\mathrm{P}$ factor. This may be because the P1 group may frequently show curability B. At our institution, and as reported by studies at other institutions, survival was considerably longer in the curability B group compared with the curability $\mathrm{C}$ group [13]. In advanced gastric cancer, peritoneal metastasis is generally considered to be the most important factor associated with postoperative recurrence [14]. Other institutions observed no differences in survival rates among P1, P2, and P3 [8]. At our institution, survival was significantly longer in the $\mathrm{P} 1$ group than in the $\mathrm{P} 2$ or $\mathrm{P} 3$ group $(P=0.0057)$. In stage IV gastric cancer, studies have shown the effects of total visceral resection in the left upper abdomen, proposed by Oyama et al. [15] and some studies suggested improvement in prognosis brought about by total visceral resection in the left upper abdomen + Appleby operation, proposed by Furukawa et al. [16]. We have used a technique in which P1 lesions are resected en bloc together with the epiploic foramens, such as by combined resection of the spleen and pancreatic tail. In P1, en-bloc resection of peritoneal metastatic lesions, as far as circumstances permit, appears to be important in prolonging survival.

Liver metastasis of gastric cancer is generally treated by hepatectomy, when possible. Patients not indicated for hepatectomy are treated by hepatic arterial infusion therapy after the placement of a reservoir, transcatheter arterial chemoembolization (TACE), systemic chemotherapy, microwave coagulation therapy (MCT), or immunotherapy. The prognosis of liver metastasis of gastric cancer has been improved by these methods, but it is still poor because single liver metastasis is infrequent compared with that seen with colorectal cancer. At our department, methods such as hepatic arterial infusion therapy and MCT have been used, but no marked improvement in the survival rate has been observed. Therefore, there was no difference in survival according to degree of liver metastasis in this study. Further studies are necessary.

Nor were any significant differences observed according to degree of lymph node metastasis, but the survival rate decreased slightly with progression of lymph node metastasis. In general gastric cancer, the prognosis becomes poorer with the degree of lymph 
node metastasis. However, in stage IV gastric cancer, survival was not affected by progression of lymph node metastasis alone, partly because of the involvement of other intraperitoneal metastasis factors. Multivariate analysis also revealed that lymph node metastasis was not an important prognostic factor. Therefore, no significant differences were observed. The slightly poorer prognosis with progression of lymph node metastasis may be due to progression of the disease stage and an increase in the tumor volume.

As described above, survival was considerably longer in the curability B group compared with the curability $\mathrm{C}$ group at our institution, as has also been reported at other institutions [13]. According to the first English edition of the Japanese classification of gastric carcinoma [3], curability B denotes no macroscopic residual cancer. This difference in the absolute volume of the residual tumor between curability B and curability C, with definite residual cancer, may have been responsible for the difference in the survival rate.

Lymph node dissection for stage IV gastric cancer has been reported to be effective by some authors, but has been reported to be ineffective by others. Aiko and Aridome [17] evaluated the usefulness of D2 or greater lymph node dissection for stage IV gastric cancer according to constituting factors and observed significant prolongation of survival for T4, N3/N4; they suggested that D2 or greater lymph node dissection should be performed, in principle, for stage IV gastric cancer. We compared survival according to the degree of lymph node dissection (D0, D1; D2, D3, D4) and found a significantly lower survival rate in the slight dissection group (D0, D1) than in the marked dissection group (D2, D3, D4) $(P=0.0006)$. Evaluation in the curability $\mathrm{C}$ group also showed a significantly lower survival rate in the slight lymph node dissection group. Most cases of stage IV gastric cancer are considerably advanced at the time of laparotomy. However, D2 or greater lymph node dissection is useful for reducing the absolute number of tumor cells in the body and for prolonging survival not only in curability B patients but also in curability $\mathrm{C}$ patients with definite residual cancer.

No significant differences were observed in survival between the two gastrectomy methods. In operations for gastric cancer with lymph node dissection, total gastrectomy is more frequently performed than subtotal gastrectomy for advanced cancer, and the prognosis is slightly poorer after total gastrectomy. However, in stage IV cancer, when curability B cannot be expected, subtotal gastrectomy is often used, in terms of the postoperative quality of life, and the operative method is not always determined based on lymph node dissection. This may be associated with our finding of the absence of differences in survival between the gastrectomy methods.
There are various opinions about chemotherapy, a major method in pre- and postoperative multidisciplinary treatment [18]. In stage III gastric cancer, multidisciplinary treatment such as chemotherapy, in addition to operation, is considered to improve the prognosis. However, operation also has limits for stage IV cancer. Although much is expected of multidisciplinary treatment, methods differ among institutions, and the methods and usefulness of multidisciplinary treatment have not yet been established. If we consider the patient's quality of life, we must take into account the pain caused by chemotherapy in relation to the lack of established effects, as well considering that the patient is kept in hospital, whereas, without the chemotherapy, in the remaining survival period, the patient may lead a meaningful life. Therefore, we evaluated various parameters to identify those that are useful for classification of patients into those indicated for chemotherapy because life-prolonging effects are expected and those indicated for early discharge without the pain caused by chemotherapy, because the life-prolonging effects are not expected.

In the curability B group, significantly longer survival was observed in the postoperative chemotherapy group than in the non-postoperative chemotherapy group. However, in the curability $\mathrm{C}$ group, no significant difference was observed between the two groups. Patients with curability $\mathrm{B}$ achieved by operation may be indicated for postoperative chemotherapy, but further evaluation is necessary for those with curability $\mathrm{C}$.

Most of the individual factors in gastric cancer evaluated in this study are closely inter-related. It is difficult to predict survival in all patients with gastric cancer based on only one prognostic factor. Therefore, the prognostic weights of various factors have been studied [1,2,4,6-8,9-12]. Recent studies of prognosis by multivariate analysis have often used Cox's multivariate analysis type life table model. Maruyama [4] performed Cox's multivariate analysis and observed the highest risk ratio for depth of invasion, followed, in descending order, by lymph node metastasis, macroscopic type, location, and histological type; he identified these factors as prognostic factors. His study, as well as most other studies, performed analysis in patients with gastric cancer in all stages [1,2,6,7,9-12]. Studies involving all patients who underwent gastrectomy as subjects have suggested hematogenous metastasis and peritoneal metastasis as important prognostic factors. In studies with patients who underwent curative operation as subjects, depth of invasion and lymph node metastasis were often identified as important prognostic factors. In this present study, done only in patients with stage IV gastric cancer, the effects of individual factors on survival could be more accurately clarified. Maruyama [4] evaluated 25 factors, based on three types of 
prognostic factors for gastric cancer (host, tumor, and treatment). Because stage IV cancer alone was evaluated in our present study, only 15 factors were analyzed, because of the number of patients. Multivariate analysis showed a high $\mathrm{RH}$, i.e., a high prognostic weight, for treatment factors (lymph node dissection, curability). A reduction in the absolute number of cancer cells in the body by operation appears to prolong survival. Therefore, differences in the prognosis of stage IV gastric cancer according to the treatment method are rational. Concerning peritoneal metastasis showing the third highest $\mathrm{RH}$, survival was prolonged in the P1 group, in whom curability B is expected. This may also be due to a reduction in cancer cells. However, lymphatic invasion and venous invasion showed the fourth highest prognostic weight. The finding that vessel invasion factors had a higher weight than the factors determining stage IV (liver metastasis, lymph node metastasis) is very interesting, because the malignancy of cancer affects prognosis, even in advanced cancer.

\section{Conclusion}

We evaluated prognostic factors in stage IV gastric cancer by univariate and multivariate analyses, with the following results: (1) Univariate analysis revealed significant differences in survival according to degree of lymphatic invasion and venous invasion; peritoneal metastasis; curability; degree of lymph node dissection; and between the presence or absence of postoperative chemotherapy. (2) Multivariate analysis showed the highest prognostic weight for lymph node dissection, followed, in descending order, by curability, peritoneal metastasis, lymphatic invasion, and venous invasion. (3) In the curability B group, postoperative chemotherapy was important. (4) In the curability C group, D2 or greater lymph node dissection resulted in prolongation of survival.

\section{References}

1. Bandoh $\mathrm{T}$, Toyoshima $\mathrm{H}$, Isoyama $\mathrm{T}$. Evaluation of prognostic factors of advanced gastric cancer by Cox proportional hazard model (in Japanese with English abstract). Jpn J Gastroenterol Surg 1993;26:2567-71.

2. Okajima K. Prognostic factors of gastric cancer - a study by univariate and multivariate analysis (in Japanese with English abstract). Jpn J Gastroenterol Surg 1997;30:700-11.
3. Japanese Research Society for Gastric Cancer. Japanese classification of gastric carcinoma. 1st English ed. Tokyo: Kanehara;1995.

4. Maruyama K. The most important prognostic factors for gastric cancer patients: a study using univariate and multivariate analysis. Scand J Gastroenterol 1987;22:63-8.

5. Sano T, Sasako M, Kinoshita T, Maruyama K. Recurrence of early gastric cancer: follow-up of 1475 patients and review of the Japanese literature. Cancer 1993;72:3174-8.

6. Nakajima T, Ota K, Ishihara S, Wakabayashi K, Nishi M. Multivariate analysis of prognostic factors in gastric cancer with Cox's proportional hazard model (in Japanese with English abstract). Jpn J Cancer Chemother 1994;21:1768-74.

7. Baba H, Korenaga D, Okamura T, Saito A, Sugimachi K. Prognostic factors in gastric cancer with serosal invasion. Arch Surg 1989;124:1061-4.

8. Japanese Research Society for Gastric Cancer. Gastric cancer in Japan (in Japanese). Tokyo: Kanehara;1996. p 703-21.

9. Bando E, Takeshita Y, Yoshimoro K, Minatoya G, Yoshimitsu Y, Isobe Y. Evaluation of peritoneal lavage cytology in gastric cancer operation: a study on the prognostic significance analyzed by Cox proportional hazard model (in Japanese with English abstract). Jpn J Gastroenterol Surg 1998;31:929-34.

10. Haraguchi M, Watanabe A, Moriguchi S, Korenaga D, Maehara $\mathrm{Y}$, Okamura T, et al. DNA ploidy is a major prognostic factor in advanced gastric carcinoma: univariate and multivariate analysis. Surgery 1991;110:814-9.

11. Setala LP, Kosma VM, Marin S, Lipponen PK, Eskelinen MJ, Syrjanen KJ, et al. Prognostic factors in gastric cancer: the value of vascular invasion, mitotic rate and lymphoplasmacytic infiltration. Br J Cancer 1996;74:766-72.

12. Moriguchi S, Hayashi Y, Nose Y, Maehara Y, Korenaga D, Sugimachi K. A comparison of the logistic regression and the Cox proportional hazard models in retrospective studies on the prognosis of patients with gastric cancer. J Surg Oncol 1993;52:9-13.

13. Sano T, Katai H, Sasako M, Maruyama K. Treatment strategy for Stage IV gastric cancer in terms of survival benefit (in Japanese). Shoukaki Geka (Gastroenterological Surgery) 1997;20:1337-42.

14. Tateishi M, Ichiyoshi Y, Kawano T, Toda T, Minamisono Y, Nagasaki S. Recurrent pattern of digestive tract carcinoma in the Japanese: comparison of gastric cancer to colon cancer. Int Surg 1995:80:41-4.

15. Oyama S, Nakajima T, Ota K, Ishihara S, Wakabayashi K, Nishi M. Left upper abdominal evisceration for advanced gastric cancer (in Japanese with English abstract). Jpn J Cancer Chemother 1994;21:1781-6.

16. Furukawa H, Hiratsuka M, Iwanaga T, Imaoka S, Ishikawa $\mathrm{O}$, Kabuto $\mathrm{T}$, et al. Extended surgery - left upper abdominal exenteration plus Appleby's method - for type 4 gastric carcinoma. Ann Surg Oncol 1997;4:209-14.

17. Aiko T, Aridome K. Lymphadenectomy for gastric cancer with Stage IV (in Japanese). Shoukaki Geka (Gastroenterological Surgery) 1997;20:1343-9.

18. Kiyohashi A, Kurihara M, Yoshida S, Ohkubo T, Suga S. Antitumor effect and survival benefit of chemotherapy for unresectable advanced gastric cancer. Jpn J Clin Oncol 1993; $23: 41-5$. 